\title{
LOS REVERENCIALES EN LAS LENGUAS MAYAS
}

Otto Schumann G. IIA-UNAM

Todos los materiales empleados en este estudio constituyen parte de la recopilación de datos lingüísticos que he hecho sobre diversas lenguas mayas. Cabe aclarar que cuando contrasto elementos de lenguas indígenas con la lengua española, me baso en la variante coloquial, hablada, de esta última, y no me refiero ni al estilo literario, ni tampoco al lenguaje impreso. De tal manera que empleo tú, vos, $U d$. según el uso en Guatemala.

Para hablar de los reverenciales en las lenguas mayas hay que tener antes una visión de los pronombres libres en estas lenguas y de la manera en que ellos aparecen en su estructura.

Se puede decir que, de acuerdo con los pronombres libres en esta familia lingüística y por la forma en que éstos se construyen, estas lenguas se pueden dividir en dos grandes grupos:

A) Aquellas que forman los pronombres libres de la manera siguiente:

+ Formativo: Deíctico/Referencial + Pronominal: sufijo pronominal.

A este grupo corresponde la gran mayoría de las lenguas mayas, teniendo como ejemplos al maya yucateco, maya mopán, k'ekchi', pocomchi', pocoman, kanjobal, jacalteco, tezltal, tzotzil, chol, mam, tzutuhil, cakchiquel y chuj.

El quiché sería una subdivisión de este grupo, en donde aparece el pronominal sin formativo, representando así el pronombre libre. Estas lenguas tienen la característica de que los pronominales que están formando el pronombre libre (es decir los sufijos de este típo), se encuentran funcionando como sujetos o como objetos (es decir representan el tema de la ergatividad), y en los predicados no 
verbales representan el sujeto (construcciones donde el núcleo del predicado se llena con un adjetivo o con un nombre, etc.).

Las terceras personas singular y plural no obedecen a esta construcción ya que pueden ser incluidas en el orden de los demostrativos; en algunas lenguas las terceras personas además de su función de pronombres marcan foco o localización, y para cualquiera de éstas habría tres posibilidades.

I) él o ella próximo.

II) él o ella distante.

III) él o ella no presente, referido.

B) Las lenguas que forman el ronombre libre de la siguiente manera:

+ Pronominal: prefijo pronominal + formato: formativo.

A este grupo corresponden sólo tres lenguas de la familia: el chontal de Tabasco, el tojolabal y el huasteco.

El huasteco se colocaría en una subdivisión, ya que aquí el pronombre libre se forma por medio de una reduplicación total del pronominal.

En estas lenguas, las terceras personas sí corresponden al mismo orden que las dos primeras. Los prefijos pronominales aparecen marcando posesión en otras construcciones (no puedo asegurar esto del huasteco, pero sí de las otras dos lenguas). En este grupo no habría la posibilidad de incluir pronombres libres que marcaran foco o localización.

Lo que se puede decir que es común a todas las lenguas de esta familia, es el hecho de que todas marcan singular y plural, y que ninguna hace la diferencia entre masculinos y femeninos en lo referente a las terceras personas (como sería el caso en español o, por lo general, en las lenguas indo-europeas).

Casi todas ellas hacen una diferencia entre el inclusivo y exclusivo de la primera persona plural.

Ejemplos del grupo A:

$\begin{array}{llll}\text { k'ekchi' } & \text { kanjobal } & \text { maya yucateco } & \\ \text { laa-in } & \text { ha-in } & \text { t-en } & \text { 'yo' } \\ \text { laa-at } & \text { ha-ach } & \text { t-ech } & \text { 'tú' } \\ \text { laa-o } & \text { he-on } & \text { t-o'on } & \text { 'nosotros' } \\ \text { laa-ex } & \text { he-ex } & \text { t-e'ex } & \text { 'ustedes' }\end{array}$


No incluyo a las lenguas que pueden marcar femenino y masculino en las terceras personas, ya que éstas no lo marcan con pronombre sino con elementos clasificatorios; como sería el caso de] kanjobal:

$$
\begin{aligned}
& \text { ja' naj }=\text { él } \quad \text { traducción literal 'este varón' } \\
& \text { ja' ix }=\text { ella traducción literal 'esta hembra' }
\end{aligned}
$$

Esta forma no corresponde de ninguna manera al pronombre libre del español, como puede verse en la traducción literal; y aunque funcionalmente se aproxima a los pronombres de tercera persona en el español, no son correspondientes en tọdos los casos.

En cuanto al foco, que se marca en algunas de las lenguas mayas, en la tercera persona singular tendríamos:

mayia mopán

$\begin{array}{lll}\text { ada'a } & \text { éste } & \text { (próximo) } \\ \text { ada'o } & \text { ése } & \text { (lejano) } \\ \text { abe'e } & \text { aquél } & \text { (referido) }\end{array}$

Aún cuando se tome en consideración su similitud con los demostrativos del español, no corresponden ni en su función, ni en su equivalencia.

Por lo general, en las lenguas mayas, la ausencia de marcador se emplea para indicar a la tercera persona del singular, sobre todo en los predicados no verbales, aunque en lenguas como el chuj, esta ausencia en ocasiones marca la segunda persona del singular.

En lo referente a la inclusividad y exclusividad, ésta se marca en la primera persona plural, donde tendríamos:

\section{kanjobal}

\begin{tabular}{|c|c|}
\hline heon (inclusivo) & nosotros \\
\hline heonon (exclusivo) & \\
\hline
\end{tabular}


tojolabal

\begin{tabular}{|c|c|}
\hline ke'ntik (inclusivo) & nosotros \\
\hline ke'intikon (exclusivo) & \\
\hline
\end{tabular}

No todas las lenguas mayas marcan la diferencia entre exclusivo e inclusivo, como es el caso de las lenguas del grupo pocom.

F.jemplos del grupo B:

$\begin{array}{llll}\text { chontal de Tabasco } & \text { tojolabal } & \text { huasteco } & \\ \text { kä-ne } & \text { k-e'n-a } & \text { n-a + naa } & \text { yo } \\ \text { a-ne } & \text { aw-e'n-a } & \text { t-a + taa } & \text { tú } \\ \text { u-ne } & \text { y-e'n-a } & \text { h-a + haa } & \text { él o ella } \\ \text { kä-ne-la } & \text { k-e'n-tik } & \text { w-a + waa } & \text { nosotros } \\ \text { a-ne-la } & \text { aw-e'n-l-ex } & \text { x-a + xaa } & \text { ustedes } \\ \text { u-neh-ob } & \text { y-e'-n-l-e' } & \text { b-a + baa-tzik } & \text { ellos/as }\end{array}$

En relación a las terceras personas, las lenguas del grupo B no funcionan de la misma forma que las del $\mathrm{A}$, ya que marcan las terceras personas siguiendo el mismo patrón que las dos primeras; el tojolabal y el chontal distinguen entre exclusivo e inclusivo. $\mathrm{Me}$ resta investigar cómo funciona en el huasteco.

En las lenguas de este grupo es de importancia hacer notar que aparece un marcador de plural para todas las personas en plural, aunque no se trate del mismo marcador en todos los casos (excepto, claro está, en el huasteco). Así tenemos:

chontal

tojolabal

huasteco

-la

$-1$

$-\mathrm{ob}$

- tik

-tzik

En las lenguas del grupo pocom, que pertenecen al grupo A, aparece un marcador de plural en la segunda persona plural. El caso de estas lenguas se puede ejemplificar con el pocomam, en el que aparece el clítico take' para señalar el plural, éste, al unirse al pronombre libre de segunda persona hat, ha quedado abreviado en la forma tahat o hata, que indica la segunda persona plural. 
Después de esta visión general sobre los fenómenos que aparecen en estas lenguas en relación a los pronombres libres, podemos entrar al tema central de este trabajo: los reverenciales.

Al igual que en otras lenguas de Mesoamérica, en muchas lenguas mayas existe la tendencia a formar reverenciales con diminutivos.

No tomo en cuenta para este artículo, sin embargo, aquellas lenguas que tienen posibilidad de marcar el reverencial con alguna construcción, sino sólo aquellas que deben de hacerlo por medio de un marcador específico, ya sea al dirigirse a los ancianos o a personas que ocupen cargos de importancia dentro de su organización social.

En todas las lenguas existe la posibilidad de marcar el reverencial en formas libres, ya sean palabras o frases, pero pocas son las que lo marcan en forma definitiva, por medio de sistemas cerrados.

El tojolabal marca el reverencial anteponiendo a los nombres el elemento -ala, que marca diminutivo, éste recibe el posesivo, que, por lo general, llevan los nombres; si bien esta construcción se usa para marcar cariño de parte del hablante al oyente, cuando se usa con los ancianos marca respeto de forma constante, y no como posibilidad; ejemplos:

a-ti', tu boca / aw-ala ti', tu boquita o la boca de Ud. k-al, mi hijo (dice la mujer) / aw-ala al, 'tu hijito o su hijo de Ud. (hablándole a una mujer).

Marca respeto sólo con las segundas personas, aunque puede también usarse con las terceras de igual manera. Aparecen también en esta lengua el sufijo -ey, que sólo registré después de los términos de parentesco que se refieren al padre y a la madre, este sufijo se emplea para marcar cariño, pero también puede usarse para indicar reverencia.

El cakchiquel tiene una forma de indicar respeto, pero no !n hace siempre con diminutivo, sino que para señalarlo cuenta, en ocasiones, con léxico diferenciado, ejemplo:

tux a-bi $=$ ¿cómo te llamas? achique a-bi $=$ ¿ cómo se llama Ud.? tux $=$ ¿cómo? (familiar) achique $=$ ¿cómo? (reverencial)

Este tipo de elementos no lo tomo en cuenta, ya que, aunque corresponde en gran parte a las construcciones para marcar reverencial, enfoco este estudio en la presentación de las formas evidentes de referencia, marcadas con el pronombre. 
En las lenguas de los Cuchamatanes, los pronombres marcan a las personas, y también la exclusividad y la inclusividad; además pueden marcar reverenciales, familiares y la forma neutra. En algunos dialectos del mam existe un sufijo $-y$ que pospuesto a los marcadores de enfático o a los posesivos, indica familiaridad; sin embargo, los elementos que marcan reverencial son diferentes entre los de una variante del mam a otra, mientras que la ausencia del mismo indica la forma neutra. Los elementos que señalan familiaridad también hacen diferencia en el género, ejemplo:

Pronombres libres reverenciales familiares

\begin{tabular}{|c|c|c|c|c|}
\hline yo & hain & & & \\
\hline tú & haach & haach & nay/ya & $\begin{array}{l}\text { cha (masculino } \\
\text { txo (femenino) }\end{array}$ \\
\hline ella & ha' & (ha') & $\begin{array}{l}\text { k'o } \\
\text { nay/ya }\end{array}$ & \\
\hline
\end{tabular}

él

nosotros heon (inclusivo)

heonon (exclusivo)

ustedes heex heex nay/ya

ellos heb heb k'o

nay/ya

ellas

Como aclaré con anterioridad, existe en el kanjobal la posibilidad de indicar el género en las terceras personas, recurriendo a la construcción $+n a j$, para el masculino e $+i x$ para el femenino, que aunque tienen como base el mismo deítico que los otros pronombres, representan una frase y no un pronombre independiente, ejemplos: 
ha' ix = ella (esta hembra) heb ix = ellas (estas hembras) ha' naj = él (este varón) heb naj = ellos (estos varones)

los elementos ix y naj, son clasificadores de género, y al igual que otros clasificadores en esta lengua, forman parte de la estructura de la frase nominal, sin marcar pronombre necesariamente.

Cha y txo, pueden aparecer de forma independiente marcando segunda persona del singular, aunque también pueden ir reforzados por el sufijo - ach, que marca segunda persona singular, en su forma neutra, ejemplos:

nawal-ach txo $=$ eres 0 sos bruja

nawal-ach cha $=$ eres o sos brujo

nawal ach = eres brujo/a (forma neutra)

nawal-ach nay, nawal-ach ya = usted (reverentemente) es brujo/a tzet-sbi $=$ ¿cómo se llama él o ella?, ¿cómo se llama eso?

tzet s-bi k'o = ¿cómo se llama él o ella? (con respeto)

tzet s-bi naj k'o = ¿cómo se llama él? (con respeto)

tzet sbi ix k'o = ¿cómo se llama elia? (con respeto)

tzet s-bi ix $=$ ¿cómo se llama ella o ese elemento femenino?

tzet s-bi naj = ¿cómo se llama él o ese elemento masculino?

tzet a-bi $=\dot{i}$ cómo te llamas? (forma neutra)

tzet a-bi nay, tzet a-bi ya $=\dot{¿}$ cómo se llama usted? (reverente)

Los pronominales de segundas y terceras personas, pueden is acompañados indistintamente de nay o de ya para marcar reveren. cial, mientras que las terceras personas pueden llevar el morfema $k$ 'o, que marca reverencial y tercera persona.

Los elementos naj e $i x$, indican masculino y femenino respectivamente. En estas lenguas pueden llevar indicador de género las personas, los animales, algunos elementos vegetales y otros meramente culturales; es decir, no marcan sexo, sino género gramaticalmente condicionado por la cultura.

Lo importante del trabajo radica en que hace notar la dificultad de la traducción en estos casos, aparentemente tan simples, pero que, como puede apreciarse, serían imposibles de traducir sin usar descripciones en el español; pues una traducción directa sería inexacta.

Es importante hacer notar que el patrón presentado aquí por medio del ejemplo del kanjobal, hacer ver que se trata de una lengua que pertenece a una sociedad históricamente muy estratificada, ya que así lo indican los pronombres que presenta. Es evidente que una 
sociedad puede tener una división social muy bien delimitada en clases, sin llegar a presentar evidencia específica en la lengua, como sería el caso del inglés, que señala a la segunda persona singular y plural con un solo elemento (en su forma colonial) y no distingue entre familiar, reverencial,, neutro y plural. Sin embargo, los países de habla inglesa están constituidos por sociedades en las cuales las clases se encuentran muy bien determinadas. En el caso de que la lengua indique una situación de estratificación, corresponde necesaria. mente a una división marcada; como en el caso del kanjobal, que si bien en la actualidad no se ve muy claramente, contamos, además de los datos históricos, con otros datos arqueológicos que pueden aplicarse de manera general a los grupos mayas y podemos constatarlo con el estudio de la iconografía maya. Si se toma en cuenta que se trata de sociedades que pasaron de ser independientes a dominadas mediante conquista, lo que implicó el exterminio de su grupo culto y su clase dominante, en esas circunstancias de sometimiento, no es posible desarrollar sistemas pronominales tan complejos; más bien, el hecho de que varias lenguas mayas conserven estos rasgos, implica que se trata de elementos retenidos del desarrollo histórico previo de estos pueblos. Los ejemplos que aparecen en este artículo hacen evidente la relación entre lengua y sociedad, y confirman así datos históricos y arqueológicos que se tienen de los grupos mayas.

Con los materiales aquí presentados, se hace obvio que resulta difícil expresar en alguna lengua europea la diferencia entre grados de familiaridad, de reverencia y la otra forma, que llamo neutra, en las distintas maneras de marcar la segunda persona singular. La distincićn entre masculino y femenino que presenta este pronombre en su forma familiar, puede ser traducida al español por medio de una frase, pero sería muy difícil marcar la diferencia entre los tres grados mencionados y su uso, ya que esto está dado culturalmente.

Seleccioné al kanjobal (en su variante de San Miguel Acatán) para ejemplificar los pronombres, por ser la lengua que reúne la mayor cantidad de casos posibles, aunque sin llegar a agotarlos; creo que una investigación que abarque otros sociolectos (como el religioso..., etc.), ampliaría las posibilidades.

Notas:

1) Los elementos gráficos que deben ser aclarados, son los siguientes: 
$\mathrm{x}=$ representa la fricativa alveopalatal sorda. En el kanjobal marca la misma fricativa con retroflexión.

k' = la oclusiva velar sorda glotalizada.

$\mathrm{k}$ = en pocomam señala la oclusiva posvelar sorda glotalizada.

$\mathrm{tx}_{\mathrm{x}}=$ africada alveopalatal sorda, retrofleja.

No me es posible usar ninguno de los alfabetos fonéticos internacionales, por la ausencia de tipos que los contengan en las tipografías usadas por la U.N.A.M.

II) Las formas de marcar la segunda persona singular, y su uso en el español coloquial de Guatemala, a grandes rasgos, sería la siguiente:

Usted, marca respeto, es empleado entre desconocidos en un primer encuentro, su uso, de esta manera, se extiende desde niños hasta ancianos; se utiliza para dirigirse a personas mayores de edad en relación al hablante (no considero el caso cuando es usado entre parejas de casados o de novios para expresar cariño).

$T u$, forma familiar usada por lo general en la clase media, entre mujeres; una mujer de esta clase o de clase media baja puede usar esta forma al dirigirse a un varón; su uso es poco frecuente. Entre varones es permitido solamente cuando se usa con extranjeros procedentes de países de habla española no se emplea el vos; el uso del tú entre varones (centroamericanos o del país), se asocia por lo general con conducta homosexual.

Vos es la forma familiar más usada, se extiende a todas las clases sociales, aunque en las clases medias su uso es más frecuente entre personas del mismo sexo.

El uso de las formas familiares con personas desconocidas, tal y como se da en el área rural de México y Guatemala, denota una jerarquía social superior del hablante con relación al oyente (el vos en Guatemala y partes de México, y el tú en casi todo el territorio mexicano, para dirigirse a las clases desposeídas). De ahí que los indígenas con menor grado de biiingüismo, sólo conocen del español las formas familiares, que son las que ellos reciben.

\section{REFERENCIA BIBLIOGRÅFICA}

Arzápalo Marín Ramón

1973 Das Pronominalsystem des Yucatekischen, Struktura-Band G., München, R.F.A. 
Pronombres Libres

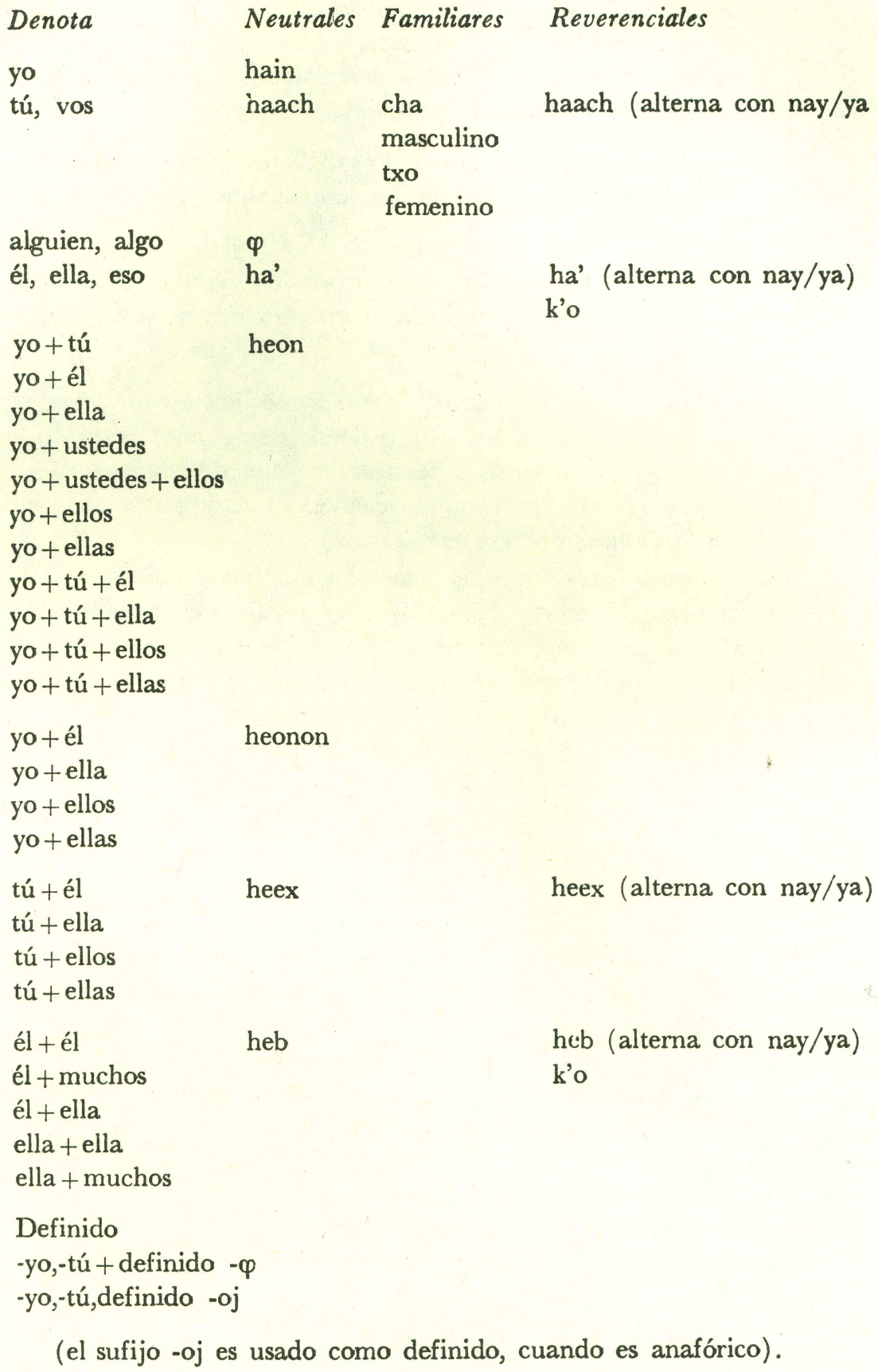

yo +él heonon

ha' (alterna con nay/ya) k'o

heon

heb (alterna con nay/ya) k'o 